\title{
Pulmonary tuberculosis with acute respiratory failure: yet to be conquered
}

\section{To the Editors:}

In the current issue of the European Respiratory Journal, KIM et al. [1] nicely discuss a scarcely reported association between pulmonary tuberculosis (TB) and acute respiratory failure (ARF). They conclude that this tuberculosis-related critical condition has a high mortality rate and is associated with risk factors predicting poor outcome. However, certain issues in the study require clarification and comprehensive discussion, so that this condition can be properly understood and managed.

The diagnosis in the study by KIM et al. [1] was confirmed by sputum smear and/or culture for acid-fast bacilli in 80 out of 90 patients; the mean interval from hospital admission to commencement of anti-TB treatment was $5.0 \pm 7.0$ and $2.8 \pm 2.5$ days in the TB pneumonia and miliary TB groups, respectively. Culture for AFB takes 6-8 weeks to be interpretable, so treatment based on culture was probably excluded in this study.

KIM et al. [1] do not mention anti-TB treatment regimens administered to the patients. Treatment has been considered to be a vital factor affecting patients' outcome in pulmonary TB [2]. Anti-TB drug regimens, total duration of treatment, method of administration (under direct observation) and proper monitoring are key treatment-related factors that should be evaluated in all TB patients with or without ARF. Treatment factor should also be discussed in detail in the study of KIM et al. [1], as the research comprised retrospective data from over 17 yrs (from 1989), during which time significant changes in the treatment of $\mathrm{TB}$ have occurred, the most important being implementation of directly observed therapy, short course.

KIM et al. [1] found advanced age and nonuse of steroids to be important factors influencing survival of TB patients with ARF. Due to a decrease in immunological response and nutritional deficiencies, old age is associated with increased incidence of infections, cancer etc. [3]. In reality, advanced age is a confounding prognostic factor in all infections, not $\mathrm{TB}$ in particular. Corticosteroid use in TB is still an area of active research and has associated advantages and pitfalls. Adjunct therapy with steroids, in conjunction with anti-TB drugs, may be cautiously used in early phase treatment in selected patients with severe forms of pulmonary and extra-pulmonary TB [4, 5]. However, every effort should be made to prevent their irrational use as they may cause a significant increase in incidence of TB [6]. Moreover, their role is not standardised in any TB treatment guidelines across the globe.

In the study by KIM et al. [1], the hospital mortality rate for patients with respiratory failure due to pulmonary TB was found to be $65.6 \%$; more than twice that found in other studies for patients with respiratory failure due to severe pneumonia [7]. It is therefore crucial to detect all relevant risk factors (including multi-drug resistance, treatment delay, multi-organ involvement etc.) in such patients and manage them without delay.

As tuberculosis is endemic in many parts of the world, this critical condition warrants further prospective evaluation for better management.

\section{Aggarwal and P.R. Mohapatra}

Dept of Pulmonary Medicine, Government Medical College and Hospital, Chandigarh, India.

\section{STATEMENT OF INTEREST}

None declared.

\section{REFERENCES}

1 Kim YJ, Pack KM, Jeong E, et al. Pulmonary tuberculosis with acute respiratory failure. Eur Respir J 2008; 32: 1624-1629.

2 Pablos-Mendez A, Sterling TR, Frieden TR. The relationship between delayed or incomplete treatment and all-cause mortality in patients with tuberculosis. JAMA 1996; 276: 1223-1228.

3 Chandra RK. Nutritional regulation of immunity and risk of infection in old age. Immunology 1989; 67: 141-147.

4 Smego RA, Ahmed N. A systematic review of the adjunctive use of systemic corticosteroids for pulmonary tuberculosis. Int J Tuberc Lung Dis 2003; 7: 208-213.

5 Alzeer AH, FitzGerald JM. Corticosteroids and tuberculosis: risks and use as adjunct therapy. Tuber Lung Dis 1993; 74: 6-11.

6 Agrawal PN, Gupta D, Aggarwal AN, Behera D. Incidence of tuberculosis among patients receiving treatment with oral corticosteroids. J Assoc Physicians India 2000; 48: 881-884.

7 Penner C, Roberts D, Kunimoto D, Manfreda J, Long R. Tuberculosis as a primary cause of respiratory failure requiring mechanical ventilation. Am J Respir Crit Care Med 1995; 151: 867-872.

DOI: $10.1183 / 09031936.00117308$

\section{From the authors:}

We are grateful to D. Aggarwal and P.R. Mohapatra for their thoughtful comments.

Regarding the importance of the information on anti-tuberculous (TB) medication, D. Aggarwal's opinion is definitely correct. In Korea, a 6-month short course of chemotherapy (with four drugs: isoniazid; rifampicin; ethambutol; and pyrazinamide) was adopted in the mid-1980s in private sector hospitals and in 1990 in healthcare clinics (public sector) [1]. Hence, in our study in the present issue [2], all patients, except two multidrug-resistant tuberculosis patients, were started on first-line anti-TB treatment; no changes were made in the 
treatment provided for patients with TB, even though the quality of critical care has improved dramatically since the beginning of this study [2]. In Korea, directly observed therapy (DOT) is not routinely followed [1]. Instead, daily therapy is given, even during the continuation phase, in almost $100 \%$ of patients. Ethambutol is usually prescribed for the entire 6 months. These are probably the reasons why TB has been controlled successfully in Korea, despite the fact that DOT has not been adopted. The prevalence of TB dropped dramatically from $5.1 \%$ in 1965 to $1.0 \%$ in 1995 [3]. In our present study [2], because all patients were supported by mechanical ventilation, anti-TB drugs were taken via nasogastric tube or gastrostomy tube, and it was not possible to skip medications.

As D. Aggarwal commented, the mortality rate was very high in this study. However, drug resistance, especially multidrugresistance, was not an important risk factor for mortality because only 2 out of 90 patients had multidrug-resistant tuberculosis. Even though old age was revealed to be a poor prognostic factor in patients with tuberculosis with respiratory failure who required mechanical ventilation, as D. Aggarwal mentioned, old age is already a well-known poor prognostic factor in several diseases. The most important finding in this study is that corticosteroid use may reduce mortality in patients with severe pulmonary tuberculosis with respiratory failure who require mechanical ventilation. However, because of the inherent limitations of this retrospective study, investigators cannot conclude that steroids are beneficial in reducing mortality in these patients. A prospective randomised study should be conducted to further explore this topic.

\section{Y.J. Kim and T.S. Shim}

Division of Pulmonary and Critical Care Medicine, University of Ulsan College of Medicine, Asan Medical Center, Seoul, South Korea.

\section{STATEMENT OF INTEREST}

None declared.

\section{REFERENCES}

1 Seung KJ, Bai GH, Kim SJ, Lew WJ, Park SK, Kim JY. The treatment of tuberculosis in South Korea. Int J Tuberc Lung Dis 2003; 7: 912-919.

2 Kim YJ, Pack KM, Jeong E, et al. Pulmonary tuberculosis with acute respiratory failure. Eur Respir J 2008; 32: 1624-1629.

3 Hong YP, Kim SJ, Lew WJ, Lee EK, Han YC. The seventh nationwide tuberculosis prevalence survey in Korea, 1995. Int J Tuberc Lung Dis 1998; 2: 27-36.

DOI: $10.1183 / 09031936.00125708$

\section{High incidence of sputum smear negative tuberculosis during HAART in Burkina Faso}

\section{To the Editors:}

Tuberculosis (TB) and HIV co-infection is a public health priority in sub-Saharan Africa, where TB is the leading cause of death among HIV infected patients and the first manifestation of the HIV infection. [1-3]. An unprecedented global effort allowed increasing access to antiretroviral treatment in Africa, where $>2$ million persons received highly active antiretroviral therapy (HAART) at the end of 2007 [1].

There is evidence that HAART reduces the risk of TB, in both industrialised [4] and resource-limited countries in subSaharan Africa and south-east Asia [5-9]. However, there is no information on the HAART impact on the incidence of the different TB forms (sputum smear positive pulmonary (SSPPTB), sputum smear negative (SSN-PTB) and extrapulmonary (ЕРTB)).

We have measured TB incidence in a retrospective cohort of HIV infected persons who started HAART in four HIV/AIDS treatment centres in Ouagadougou, Burkina Faso. All consecutive HIV-seropositive patients aged $\geqslant 15 \mathrm{yrs}$, TB-free at HAART initiation, with a follow-up of 12 months or longer were included in the analysis. TB diagnosis was based on internationally accepted criteria [10]. Pulmonary TB (PTB) was diagnosed by microscopy (SSP-PTB) or, in the case of SSN-PTB, on algorithms requiring all the following criteria: 1) chest radiography compatible with active $\mathrm{TB} ; 2$ ) unresponsiveness to $\geqslant 1$ course of large-spectrum antibiotic; and 3) clinician's decision to prescribe a full course of anti-TB therapy. EPTB was based on the clinician's decision to prescribe a full course of anti-TB therapy on the basis of evocative clinical signs, radiological findings and biochemistry of body fluids. Routine culture for Mycobacterium tuberculosis is not available in Burkina Faso.

A cohort of 2,383 HIV-seropositive persons were followed-up for a mean period of $836.1 \pm 443.4$ days. More than a half were classified as World Health Organization (WHO) stage III or IV and $83 \%$ had a CD4 cell count of $<200$ cells $\cdot \mu L^{-1}$ at HAART initiation. A total of 70 TB cases were diagnosed, including 18 (26\%) SSP-PTB, 25 (36\%) SSN-PTB and 27 (38\%) EPTB. Among the 27 EPTB cases, the most frequent TB sites were lymph node $(12(44 \%)$ cases), pleura (5 $(18.5 \%)$ cases) and peritoneum (4 (15\%) cases).

TB incidence declined from 2.80 (95\% confidence interval (CI) $1.60-4.54)$ in the first trimester of HAART to 0.05 (95\% CI $0.01-$ 0.16 ) cases per 100 person $^{-1} \cdot \mathrm{yr}^{-1}$ for $\geqslant 12$ months after its initiation among SSN-PTB patients, from 1.40 (95\% CI 0.60-2.75) to 0.05 (95\% CI $0.01-0.16)$ cases per 100 person $^{-1} \cdot \mathrm{yr}^{-1}$ among SSP-PTB patients, and from 1.57 (95\% CI 0.72-2.99) to 0.07 (95\% CI 0.02 0.19 ) cases per 100 person $^{-1} \cdot \mathrm{yr}^{-1}$ among EPTB patients (fig. 1). 